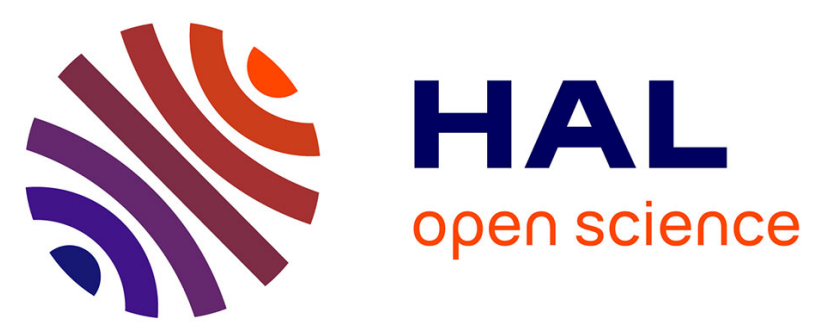

\title{
Effect of an external electric field on the coherent terahertz emission from multiple filaments in air
}

Sergey Mitryukovskiy, Yi Liu, Bernard Prade, Aurélien Houard, André Mysyrowicz

\section{- To cite this version:}

Sergey Mitryukovskiy, Yi Liu, Bernard Prade, Aurélien Houard, André Mysyrowicz. Effect of an external electric field on the coherent terahertz emission from multiple filaments in air. Applied Physics B - Laser and Optics, 2014, 117, pp.265 - 269. 10.1007/s00340-014-5830-1 . hal-01118184

\section{HAL Id: hal-01118184 \\ https://hal-ensta-paris.archives-ouvertes.fr/hal-01118184}

Submitted on 18 Feb 2015

HAL is a multi-disciplinary open access archive for the deposit and dissemination of scientific research documents, whether they are published or not. The documents may come from teaching and research institutions in France or abroad, or from public or private research centers.
L'archive ouverte pluridisciplinaire HAL, est destinée au dépôt et à la diffusion de documents scientifiques de niveau recherche, publiés ou non, émanant des établissements d'enseignement et de recherche français ou étrangers, des laboratoires publics ou privés. 


\title{
Effect of an external electric field on the coherent terahertz emission from multiple filaments in air
}

\author{
Sergey I. Mitryukovskiy, Yi Liu, Bernard Prade, Aurélien Houard and André Mysyrowicz* \\ Laboratoire d'Optique Appliquée, ENSTA ParisTech/CNRS/Ecole Polytechnique, 828, Bd des Maréchaux, Palaiseau, 91762, France \\ *Fax: +33 169319709 , E-mail: andre.mysyrowicz@ensta-paristech.fr
}

\begin{abstract}
We describe the terahertz emission from multiple filaments in air in the presence of an external electric field. A strong enhancement of the radiated terahertz energy is obtained by the combined effect of terahertz field interference and presence of a static electric field.
\end{abstract}

\section{Introduction}

Terahertz $(\mathrm{THz})$ radiation from plasma in air induced by laser pulses was first demonstrated by Hamster and co-workers about 20 years ago [1]. Since then, the THz radiation from a long plasma channel, a filament, generated by an intense femtosecond laser has attracted much attention [2-18]. For a filament formed by a single femtosecond laser pulse, the THz radiation is produced by the longitudinal plasma oscillations in the wake of the moving ionization front. These plasma oscillations are excited by the ponderomotive force of the laser pulse and are damped by electron collisions on a picosecond scale. The radiation is emitted by a dipole like structure moving at the speed of light; it is therefore a Cherenkov-like emission [3]. A laser filament as a $\mathrm{THz}$ source has several unique advantages comparing to the traditional techniques for $\mathrm{THz}$ generation including photoconductive antenna and optical rectification with electro-optical crystal. Being a femtosecond filament a $\mathrm{THz}$ source can be positioned far away from the laser system, thereby avoiding the absorption of the $\mathrm{THz}$ field by water vapor during propagation in ambient air. At the same time, high energy laser pulses can be employed for $\mathrm{THz}$ generation without damage to the emitter, which is impossible with the traditional techniques. Unfortunately, the conversion efficiency of this method is very low. It has been measured to be on the order of $10^{-9}$ [4]. However, several methods have been demonstrated to enhance the $\mathrm{THz}$ field. An enhancement of a three orders of magnitude has been reported with application of a static or optical electric field [4-11].

In our previous letter [12], we have shown that it is possible to add coherently the $\mathrm{THz}$ emission from multiple filaments, leading to an increase of the radiated $\mathrm{THz}$ energy scaling like $N^{2}$, where $N$ is the number of filaments. In this letter, we consider experimentally and theoretically the effect of a static electric field on the $\mathrm{THz}$ emission from multiple filaments.

\section{Theoretical model and calculations}

We first consider the effect theoretically. Because the longitudinal electric field merely increases the $\mathrm{THz}$ intensity without changing the radiation pattern [8] it is not necessary to consider it. We therefore focus our attention on the case of transverse electric field, where the action of the applied field leads to new emission patterns from filaments.

Our calculations are based on the Cherenkov model presented in [7]. The filament propagates along the $z$ axis (see Fig. 1(a) and Fig. 2). The THz field distribution in the far field from a single filament in the presence of a transverse electric field in the $x$ direction is described by: 


$$
\left\{\begin{array}{l}
E_{R}=0 \\
E_{\theta}=\frac{1}{4 \pi} \sqrt{\frac{\mu_{0}}{\varepsilon_{0}}} j(\omega) \frac{e^{i k R}}{R} \cos \theta \cos \varphi \frac{e^{i k L(1-\cos \theta)}-1}{1-\cos \theta} \\
E_{\varphi}=-\frac{1}{4 \pi} \sqrt{\frac{\mu_{0}}{\varepsilon_{0}}} j(\omega) \frac{e^{i k R}}{R} \sin \varphi \frac{e^{i k L(1-\cos \theta)}-1}{1-\cos \theta}
\end{array}\right.
$$

here $\omega, L, k$ represent the frequency of the considered $\mathrm{THz}$ component, the length of the filament, and the THz wave number, respectively. $R$ is the distance from the filament to the detector, $\theta$ is the polar angle and $\varphi$ is the azimuthal angle (spherical coordinates) (Fig. 2). The spectral current amplitude at frequency $\omega$ is given by

$$
j(\omega)=\frac{\varepsilon_{0} E_{S} \omega_{p e}^{2}}{\omega^{2}-\omega_{p e}^{2}+i v_{e} \omega},
$$

where $E_{s}$ is the external static electric field, $\omega_{p e}=\sqrt{e^{2} n_{e} / m_{e} \varepsilon_{0}}$ is the electron plasma frequency, $v_{e}$ is the electron collision frequency.

In the presence of an applied transverse field, the $\mathrm{THz}$ radiation pattern gradually evolves from a conical pattern to a single lobe forward oriented emission for $E>3 \mathrm{kV} / \mathrm{cm}$ [7]. In what follows, we always consider applied fields with $E>3 \mathrm{kV} / \mathrm{cm}$. The 3 dimension (3D) emission diagram for the single filament is presented in Fig. 1(a).

The Poynting vector of the THz emission scales like

$$
\bar{S}=\frac{1}{2}\left(E_{\theta} H_{\varphi}^{*}-H_{\theta}^{*} E_{\varphi}\right)=\frac{1}{32 \pi^{2}} \sqrt{\frac{\mu_{0}}{\varepsilon_{0}}} \frac{\cos ^{2} \theta \cos ^{2} \varphi+\sin ^{2} \varphi}{(1-\cos \theta)^{2}} \sin ^{2}\left(\frac{L \omega}{2 c}(1-\cos \theta)\right) \times|j(\omega)|^{2},
$$

therefore the intensity of $\mathrm{THz}$ emission depends on external electric field like $E_{S}^{2}$. In [7] the quadratic dependence of the energy of the $\mathrm{THz}$ radiation on external electric field has been verified experimentally.

One can extend the model to the case of $N$ identical filaments. The interference of the $\mathrm{THz}$ fields will give:

$$
\bar{S}^{\text {total }} \approx\left|\sum_{i=1}^{N} E_{\theta}\left(R_{i}, \theta_{i}, \varphi_{i}\right) e^{i \xi_{i}}\right|^{2}+\left|\sum_{i=1}^{N} E_{\varphi}\left(R_{i}, \theta_{i}, \varphi_{i}\right) e^{i \xi_{i}}\right|^{2},
$$

Here $\xi_{i}=\omega \tau_{i}$ is the accumulated phase difference of the $\mathrm{THz}$ fields due to the temporal delay $\tau_{\mathrm{i}}$ between the laser pulses and $R_{i}$ is the distance from the $i^{\text {th }}$ filament emitter to the detector.

The normalized calculated 3D distribution of the $\mathrm{THz}$ field from two parallel filaments is presented in Fig. 1(b). An enhancement of the THz intensity by a factor 4 is achieved comparing with the case of the single filament in the presence of the same applied field.

\section{Experimental results}


We now compare our calculations with experimental measurements. We study experimentally how the $\mathrm{THz}$ emission depends on the variations of temporal delay $\tau_{\mathrm{p}}$ and spatial separation $d$ between two parallel filaments in the presence of a transverse field. In our experiments, a femtosecond laser system delivering 40 fs pulses with energy up to $15 \mathrm{~mJ}$ at $800 \mathrm{~nm}$ was employed. The experimental set-up is presented schematically in Fig. 2. We used a MachZehnder interferometry scheme to obtain two parallel propagating pulses. The spatial separation and temporal delay of the two pulses could be controlled by a steering mirror of the interferometer and an integrated mechanic delay line. The two pulses with equal energy of 1.1 $\mathrm{mJ}$ were focused with two convex focusing lenses of $f=100 \mathrm{~cm}$ to form two $10 \mathrm{~mm}$ long parallel, laterally separated filaments in ambient air. The forward $\mathrm{THz}$ radiation was detected by a heterodyne detector working at $0.1 \mathrm{THz}$ [13]. The distance between the filaments and the detector was around $30 \mathrm{~cm}$. In our experiment the detector measures only the component in the plane of the two filaments (XOZ plane). The static electric field was applied to the ionized region transversally by placing two electrodes around the filaments. The electrodes were square copper plates with dimensions of $5 \mathrm{~cm} \times 5 \mathrm{~cm}$. We have studied two cases: when the direction of the electric field is parallel (configuration A) or perpendicular (configuration B) to the plane defined by the filaments. The applied field was $4 \mathrm{kV} / \mathrm{cm}$.

We first studied the interference of two synthesized $\left(\tau_{\mathrm{p}}=0\right) \mathrm{THz}$ fields for different spatial separations in configuration A. The results are presented in Fig. 3. Upon increase of the spatial separation $d$, the $\mathrm{THz}$ radiation pattern becomes narrower, but remains symmetric about the propagation direction of the laser pulses. Note that there is no decrease of the $\mathrm{THz}$ intensity within the range studied here. This differs from the results obtained in the absence of an electric field [12], where the THz intensity starts to decrease with the increase of the spatial separation between filaments. We attribute it to the different size of the intersection areas of two $\mathrm{THz}$ radiation patterns from each individual filament with and without external electric field. Without external electric field the $\mathrm{THz}$ emission from a filament has a narrow conical distribution, thus the overlap of the $\mathrm{THz}$ radiation patterns from two filaments is a sensitive function of separation between them. With the transverse electric field, the broader forward lobe allows larger filament separations while interference of the $\mathrm{THz}$ emissions is still present.

Next we studied the interference of two synthesized $\mathrm{THz}$ fields for different temporal delays with a fixed spatial separation $d=2 \mathrm{~mm}$ between two filaments, always in configuration A. The results are presented in Fig. 4. Upon increase of the delay $\tau_{\mathrm{p}}$, the total radiation pattern becomes asymmetric, alternatively directed to either side of the filament propagation direction. The change in direction reflects the partial destructive interference between the $\mathrm{THz}$ fields due to the phase shift induced by the delay. At the same time, the total $\mathrm{THz}$ radiation decreases. Finally $\left(\tau_{\mathrm{p}}=5.5 \mathrm{ps}\right)$, the total $\mathrm{THz}$ radiation pattern comes to near complete cancelation of the $\mathrm{THz}$ field, keeping only two small lobes in the non-intersection area. Eventually, for $\tau_{\mathrm{p}}=11 \mathrm{ps}$, the $\mathrm{THz}$ radiation recovers the value without time delay. Note that the strongest enhancement of $\mathrm{THz}$ radiation is always achieved with no temporal delay. By changing the temporal delay one can redirect the $\mathrm{THz}$ radiation along another direction that is not collinear with the laser beams. However, in most cases, the peak intensity decreases.

We have also studied the case of configuration $\mathrm{B}$, when the direction of the transverse external electric field is perpendicular to the plane of filaments and found results similar to the ones presented in Fig. 3 and Fig. 4. 
The comparison between the experiments and calculations is excellent, as can be seen in Fig. 3. We have considered theoretically the $\mathrm{THz}$ emission at a frequency of $0.1 \mathrm{THz}$, because it corresponds to the spectral component measured by our detector. However the $\mathrm{THz}$ emission from filaments peaks at higher frequencies. In Fig. 5 we show the temporal waveform of a $\mathrm{THz}$ pulse and the corresponding spectrum obtained from a $5 \mathrm{~mm}$ long filament in air in the presence of an external field of $8 \mathrm{kV} / \mathrm{cm}$. The $\mathrm{THz}$ waveform was obtained by the standard time domain $\mathrm{THz}$ spectroscopy system based on a $1 \mathrm{~mm}$ thick ZnTe crystal [14]. The THz power spectrum reaches a maximum at $0.5 \mathrm{THz}$. It is therefore instructive to calculate the emission pattern at other frequencies. In Fig. 6(b) the result for $0.5 \mathrm{THz}$ is presented for two parallel filaments separated by $500 \mu \mathrm{m}$. Such spatial separation was chosen to keep the ratio $d / \lambda$ on the same order as for $0.1 \mathrm{THz}$ ( $d$ is the spatial separation between filaments, $\lambda$ is the wavelength of the $\mathrm{THz}$ emission). This parameter is important for the constructive interference because the $\mathrm{THz}$ radiation pattern becomes narrower with higher $\mathrm{THz}$ frequency and thus the intersection area of two $\mathrm{THz}$ emission from two filaments decreasing if the separation between the filaments is fixed. For $\tau_{\mathrm{p}}=0 \mathrm{ps}$, the peak $\mathrm{THz}$ intensity is 4 times larger than for the single filament with the same external field (see Fig. 6(a)) showing again complete constructive interference when there is no time delay between the laser pulses. The behavior with time delay between the laser pulses is similar to the case presented in the Fig. 4 . It is periodic, but the period is different. The period corresponds to $1 / v_{\mathrm{d}}$, where $v_{\mathrm{d}}$ is the considered $\mathrm{THz}$ frequency.

\section{Extrapolation to the case of $N$ filaments}

We have calculated the $\mathrm{THz}$ radiation distribution further for $N=4,8,9,12$ and 16 parallel filaments organized in a square grid in the presence of an external transverse electric field. $\mathrm{THz}$ emissions from individual filaments interfere constructively with each other if there is no time delay between the filamentary pulses. The strong enhancement of $\mathrm{THz}$ emission from an array of parallel laser filaments in the presence of a transverse static electric field is achieved for all $\mathrm{THz}$ frequencies at the same time because the condition of zero time delay needed for the constructive interference applies to every frequency. The total $\mathrm{THz}$ energy is channeled toward the direction of the laser. The corresponding THz peak intensity scales like $N^{2}$, as shown in Fig. 7. The 3D distribution of the $\mathrm{THz}$ radiation for 16 parallel filaments is presented in Fig. 6(d) as an example. Its peak intensity is found to be $\sim 250$ times larger than that of a single filament in the presence of the same external field and more than $10^{5}$ higher than that of a single filament without electric field. In principle, with a larger number of filaments, the THz peak intensity may be increased further.

Note that varying of the temporal delay $\tau_{\mathrm{p}}$ leads to significant changes in the radiation diagram. For the example given in Fig. 6(c) for two parallel filaments with temporal delay $\tau_{\mathrm{p}}$ $=1 \mathrm{ps}$ the direction of the radiation at $0.5 \mathrm{THz}$ is completely different from that of the laser beams, forming two lobes on the two sides from the filament direction.

\section{Conclusion}

In summary, we have discussed the $\mathrm{THz}$ emission from femtosecond laser filaments in air under the combination of two effects: the application of static electric field and the organization of filaments into an array. An enhancement of $\mathrm{THz}$ radiation is found, scaling like $E^{2}$ in the presence of a static transverse electric field and scaling like $N^{2}$ in the presence of $N$ laser filaments organized in an array. With no temporal delay between the laser pulses forming the filament array, the enhancement is the same at every $\mathrm{THz}$ frequency, leading to strong enhancement of a broadband $\mathrm{THz}$ radiation in the forward direction. There is also a 
possibility to direct the $\mathrm{THz}$ emission along preferential directions by playing with the time delay between the laser pulses.

\section{Acknowledgements}

The research leading to these results has received funding from ANR (grant $n^{\circ}$ ANR-2010JCJC-0401-01) and from LASERLAB-EUROPE (grant agreement $n^{\circ} 284464$, EC's Seventh Framework Programme). 


\section{References}

[1] H. Hamster, A. Sullivan, S. Gordon, W. White, and R. W. Falcone: Phys. Rev. Lett. 71, 2725 (1993).

[2] C. D’Amico, A. Houard, S. Akturk, Y. Liu, J. Le Bloas, M. Franco, B. Prade, A. Couairon, V. T. Tikhonchuk, and A. Mysyrowicz: New J. Phys. 10, 013015 (2008).

[3] A. Houard, Y. Liu, A. Mysyrowicz, and B. Leriche: Appl. Phys. Lett. 91, 241105 (2007).

[4] T. Löffler, F. Jacob, and H. G. Roskos: Appl. Phys. Lett. 77, 453 (2000).

[5] D. J. Cook, and R. M. Hochstrasser: Opt. Lett. 25, 1210 (2000).

[6] M. D. Tomson, M. Kreß, T. Löffler, and H. G. Roskos: Laser Photon. Rev. 1, 349 (2007).

[7] A. Houard, Y. Liu, B. Prade, V. T. Tikhonchuk, and A. Mysyrowicz: Phys. Rev. Lett. 100, 255006 (2008).

[8] Y. Liu, A. Houard, B. Prade, A. Mysyrowicz, A. Diaw, and V. T. Tikhonchuk: Appl. Phys. Lett. 93, 051108 (2008).

[9] K. Y. Kim, A. J. Talor, J. H. Glownia, G. Rodriguez: Nature Photon. 2, 605 (2008).

[10] Y. Chen, T. Wang, C. Marceau, F. Théberge, M. Châteauneuf, J. Dubois, O. Kosareva, and S. L. Chin: Appl. Phys. Lett. 95, 101101 (2009).

[11] Y. S. You, T. I. Oh, and K. Y. Kim: Phys. Rev. Lett. 109, 183902 (2012).

[12] S. I. Mitryukovskiy, Y. Liu, B. Prade, A. Houard, and A. Mysyrowicz: Appl. Phys. Lett. 102, 221107 (2013).

[13] S. Tzortzakis, G. Méchain, G. Patalano, Y.-B. André, B. Prade, M. Franco, A. Mysyrowicz, J.-M. Munier, M. Gheudin, G. Beaudin, and P. Encrenaz: Opt. Lett. 27, 1944 (2002).

[14] Q. Wu, M. Litz, and X.-C. Zhang: Appl. Phys. Lett. 68, 2924 (1996).

[15] J. Liu, J. Dai, S. L. Chin, and X.-C. Zhang, Nature Photon. 4, 627 (2010).

[16] Y. Bai, L. Song, R. Xu, C. Li, P. Liu, Z. Zeng, Z. Zhang, H. Lu, R. Li, and Z. Xu: Phys. Rev. Lett. 108, 255004 (2012).

[17] S. Li, H. Liu, N. Huang, and Q. Sin: J. Phys. B: At. Mol. Opt. Phys. 46, 245402 (2013)

[18] L. Bergé, S. Skupin, C. Köhler, I. Babushkin, and J. Herrmann: Phys. Rev. Lett. 110, 073901 (2013). 


\section{Figure captions:}

\section{Fig. 1.}

Calculated $\mathrm{THz}$ radiation diagrams from filaments in transverse electric field parallel to the $x$ axis: (a) single filament, (b) two parallel filaments separated by $2 \mathrm{~mm}$ lying in the XOZ plane.

\section{Fig. 2.}

Experimental set up. Inset: spatial orientation of the two parallel filaments and direction of the transverse electric field used for calculations and in experiment. The filaments are separated laterally by a variable distance $d$, and $\tau_{\mathrm{p}}$ is the adjustable time delay between the two filamentary pulses.

\section{Fig. 3.}

Emission diagrams of the $\mathrm{THz}$ radiation at $0.1 \mathrm{THz}$ obtained from two filaments as a function of separation $d$. The measurements were performed in the plane of the filaments. Top row: calculations. Bottom row: experimental results. All THz intensities are normalized to the peak $\mathrm{THz}$ intensity of a single filament.

\section{Fig. 4.}

Emission diagrams evolution of the $\mathrm{THz}$ radiation in the plane of filaments, as a function of the temporal delay between the two laser pulses. The two filaments are separated by $d=2$ $\mathrm{mm}$. Top row: calculations. Bottom row: experimental results. $\mathrm{THz}$ intensities are normalized to the peak $\mathrm{THz}$ intensity of a single filament.

\section{Fig. 5.}

(a) $\mathrm{THz}$ waveform measured by a time domain $\mathrm{THz}$ spectroscopy system. (b) Corresponding spectrum of the THz field below $5 \mathrm{THz}$.

\section{Fig. 6.}

Calculated $\mathrm{THz}$ radiation diagram for the frequency of $0.5 \mathrm{THz}$ from: (a) single filament, (b) two parallel filaments separated by $500 \mu \mathrm{m}$ without time delay, (c) two parallel filaments separated by $500 \mu \mathrm{m}$ with a time delay $\tau_{\mathrm{p}}=1 \mathrm{ps}$, (d) a square matrix of 16 parallel filaments separated by $500 \mu \mathrm{m}$ without time delay.

\section{Fig. 7.}

Dependence of peak $\mathrm{THz}$ intensity on the number of filaments $N$, normalized to the $\mathrm{THz}$ intensity of a single filament subject to the same electric field $E_{\mathrm{s}}>3 \mathrm{kV} / \mathrm{cm}$. Red squares theoretical upper limit (quadratic dependence). Black circles - maximum value obtained in calculations. 
Figure 1.
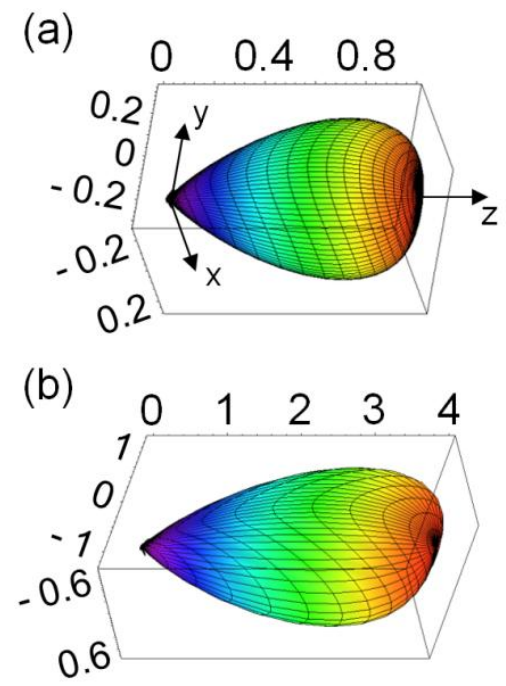

Figure 2.

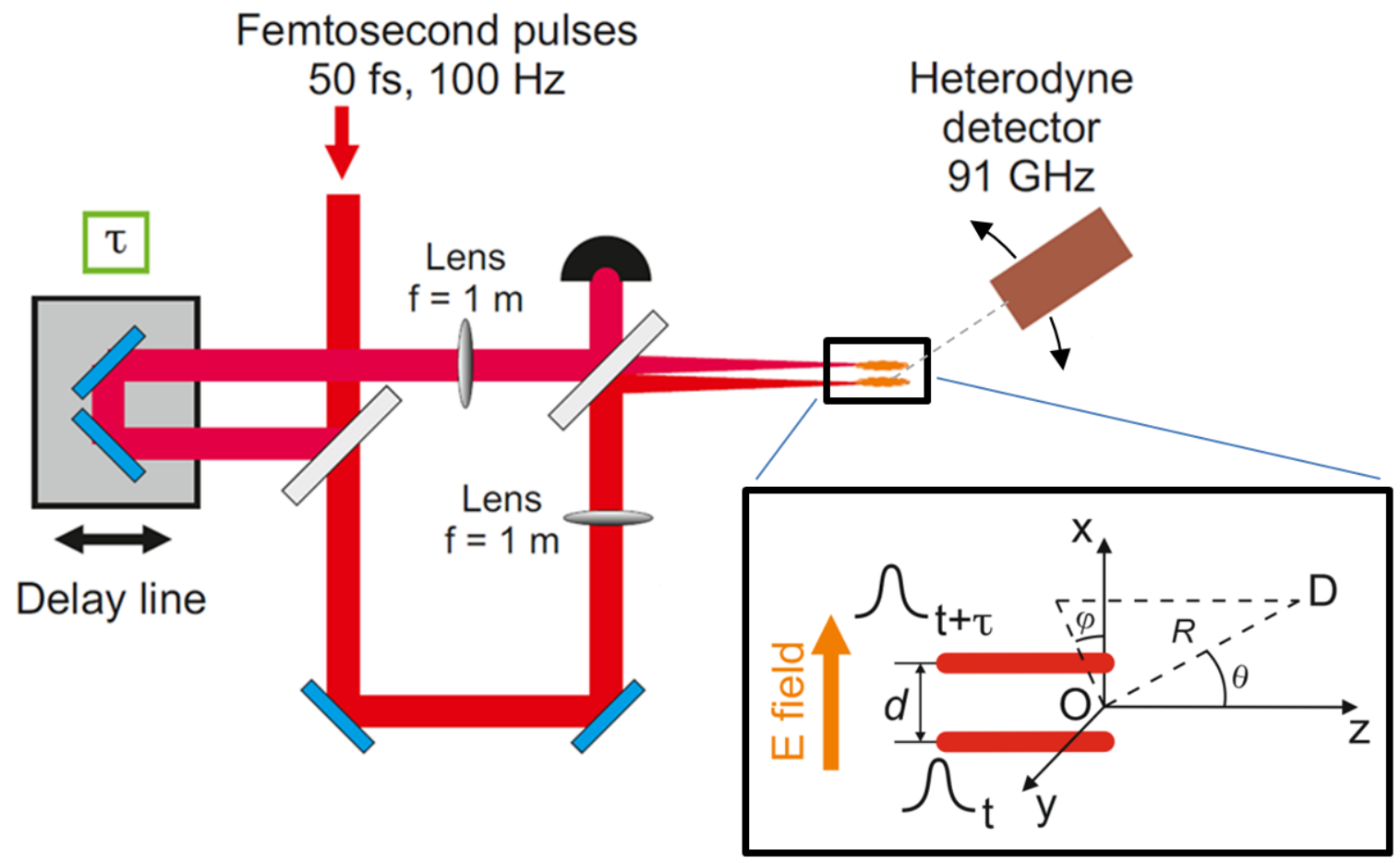


Figure 3.

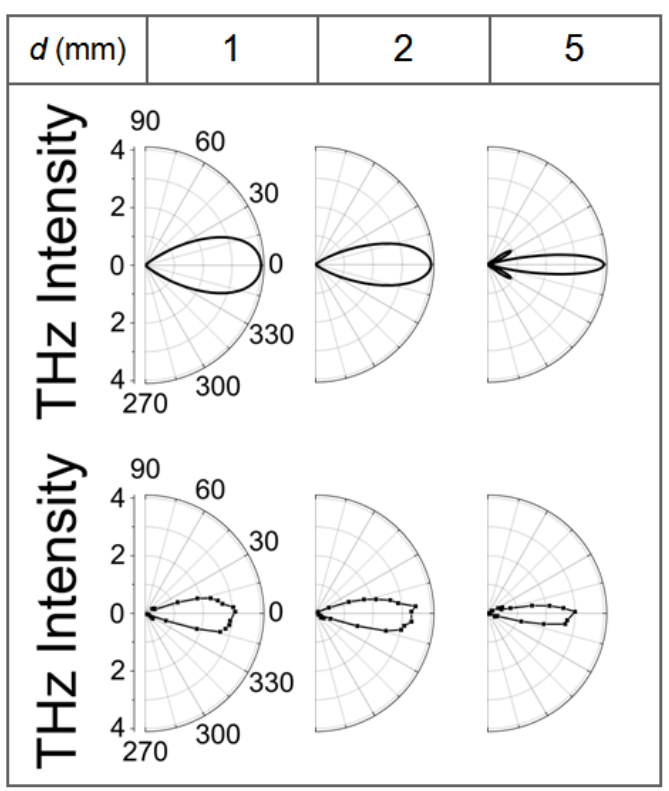

Figure 4.

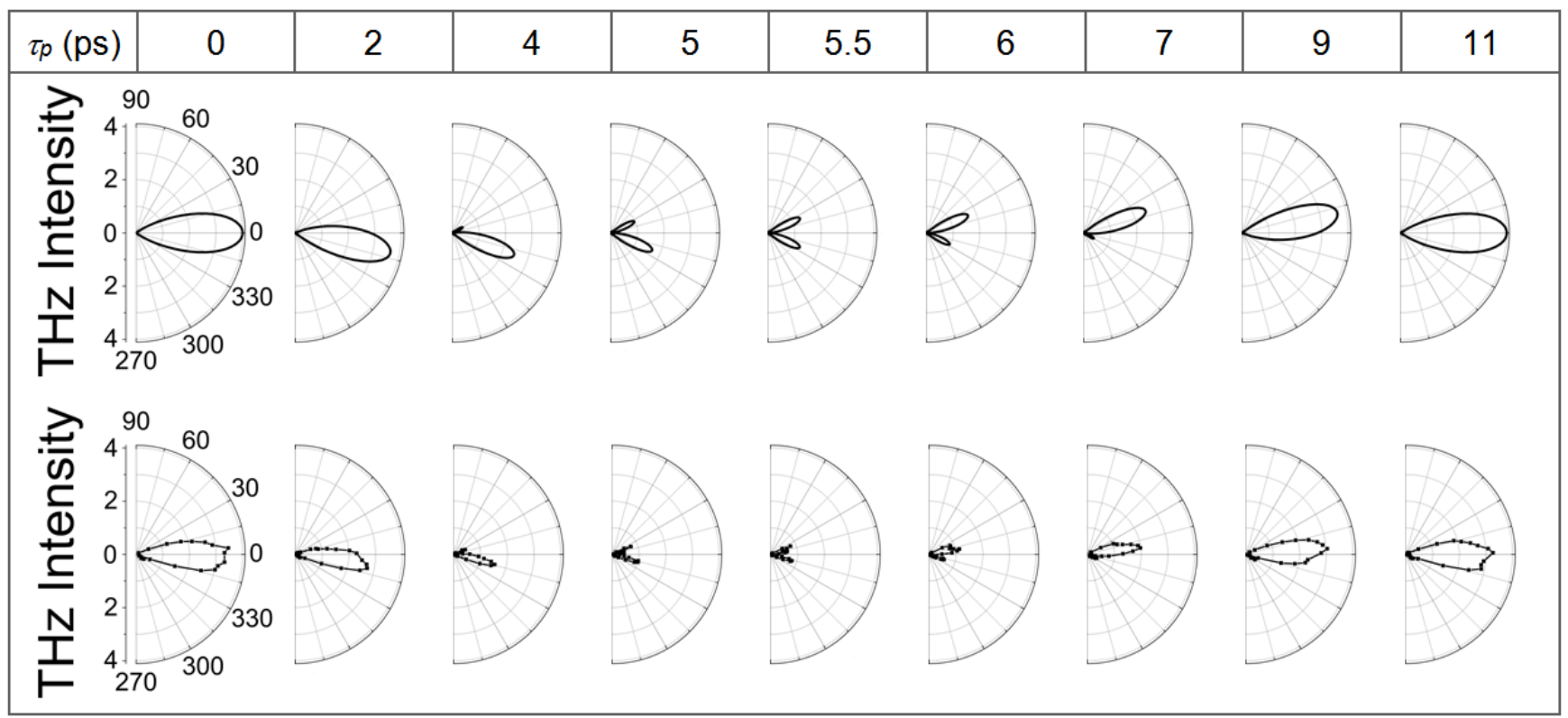


Figure 5.
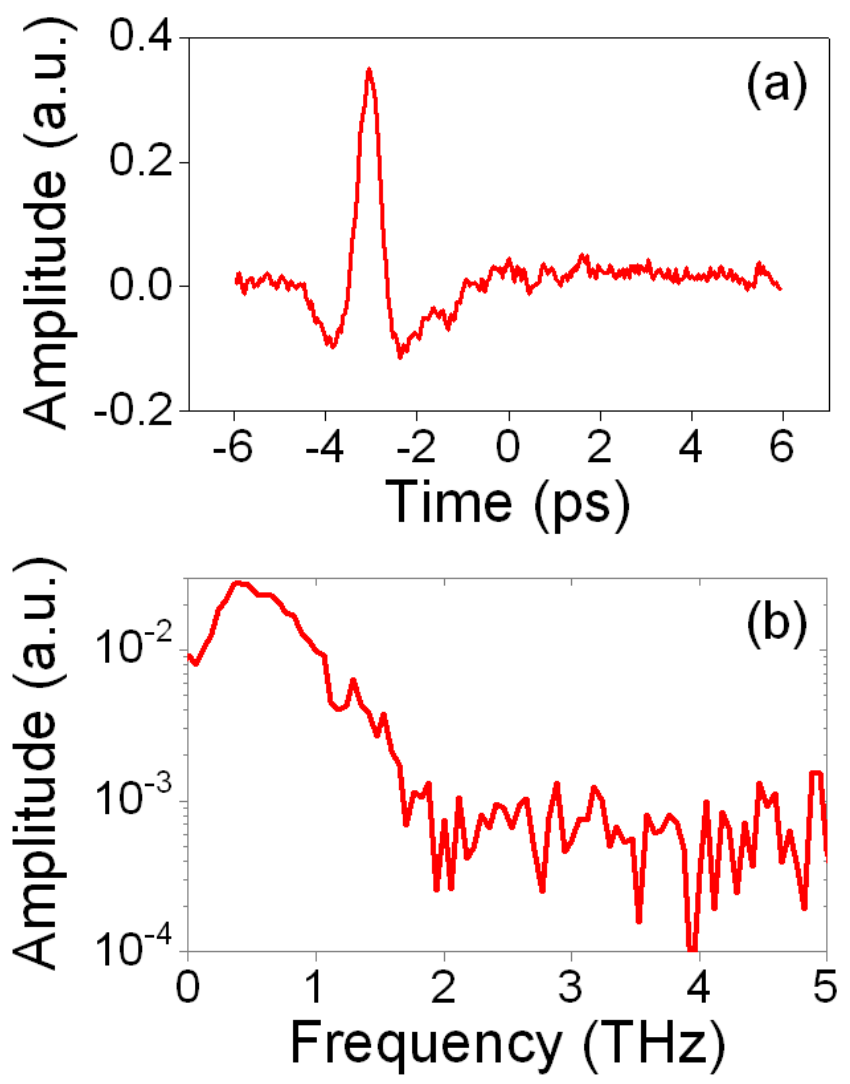
Figure 6.

(a)

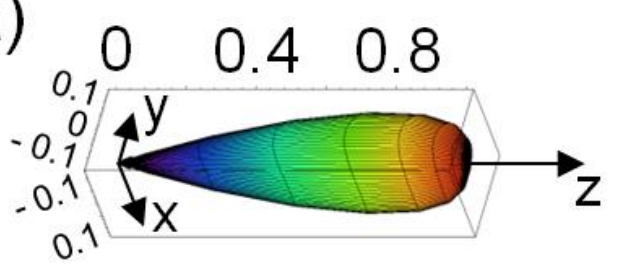

(b)

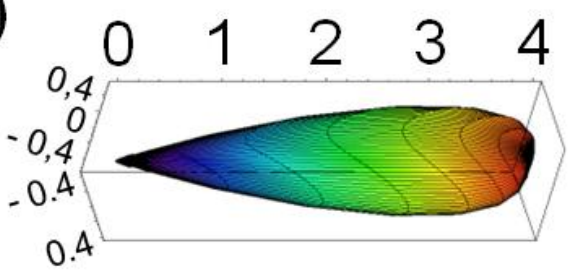

(c)

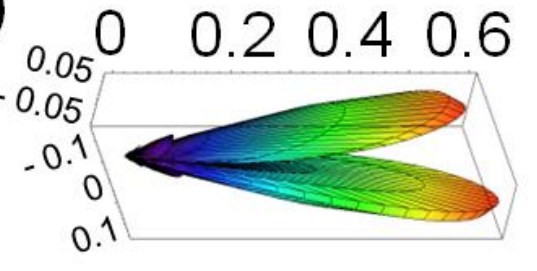

(d)

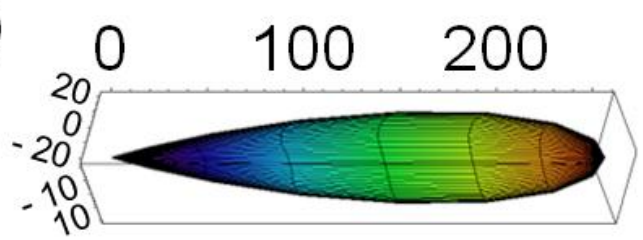

Figure 7.

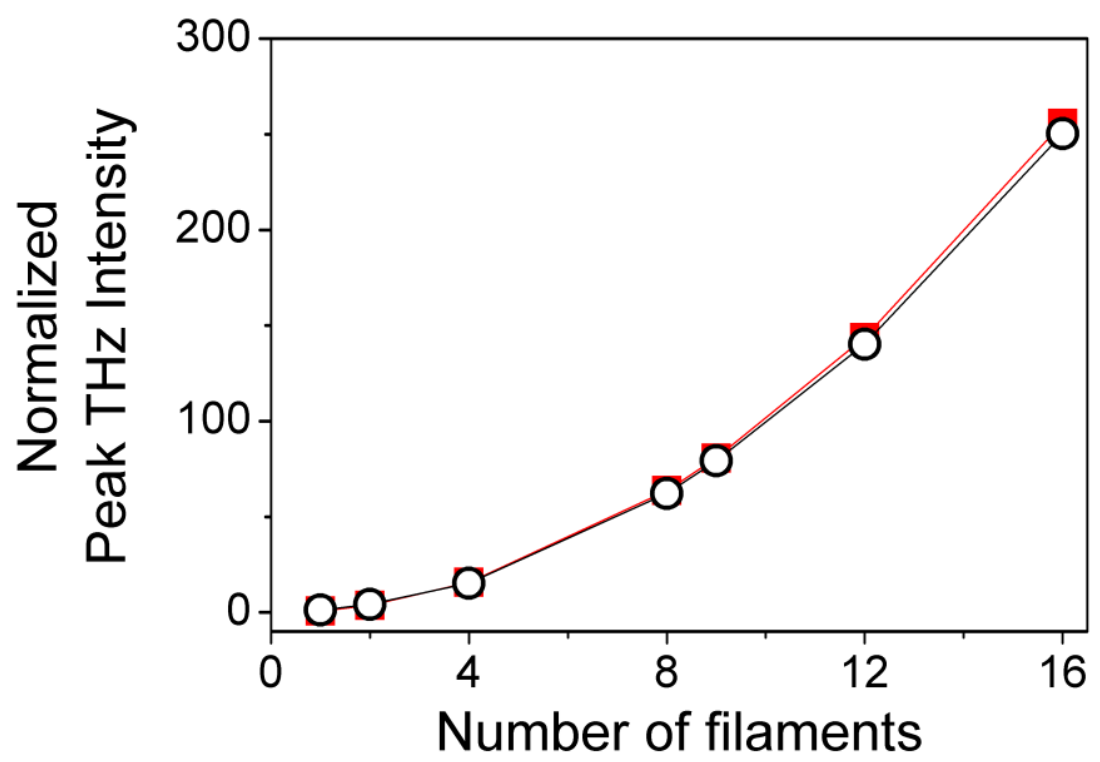

